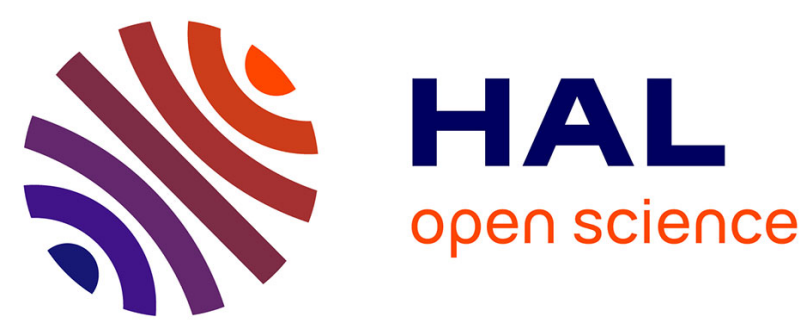

\title{
Mutations in the human kinesin Eg5 that confer resistance to monastrol and S-Trityl-L-Cysteine in tumor derived cell lines.
}

Sergey Tcherniuk, Robert van Lis, Frank Kozielski, Dimitrios A. Skoufias

\section{- To cite this version:}

Sergey Tcherniuk, Robert van Lis, Frank Kozielski, Dimitrios A. Skoufias. Mutations in the human kinesin Eg5 that confer resistance to monastrol and S-Trityl-L-Cysteine in tumor derived cell lines.. Biochemical Pharmacology, 2010, 79 (6), pp.864. 10.1016/j.bcp.2009.11.001 . hal-00556273

\section{HAL Id: hal-00556273 https://hal.science/hal-00556273}

Submitted on 16 Jan 2011

HAL is a multi-disciplinary open access archive for the deposit and dissemination of scientific research documents, whether they are published or not. The documents may come from teaching and research institutions in France or abroad, or from public or private research centers.
L'archive ouverte pluridisciplinaire HAL, est destinée au dépôt et à la diffusion de documents scientifiques de niveau recherche, publiés ou non, émanant des établissements d'enseignement et de recherche français ou étrangers, des laboratoires publics ou privés. 


\section{Accepted Manuscript}

Title: Mutations in the human kinesin Eg5 that confer resistance to monastrol and S-Trityl-L-Cysteine in tumor derived cell lines.

Authors: Sergey Tcherniuk, Robert van Lis, Frank Kozielski, Dimitrios A. Skoufias

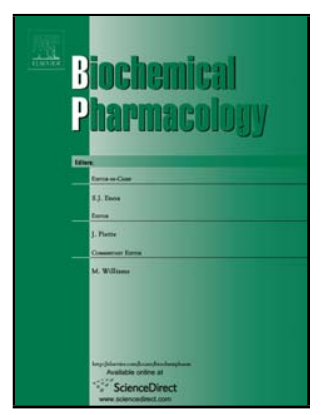

PII: S0006-2952(09)00965-4

DOI: doi:10.1016/j.bcp.2009.11.001

Reference: BCP 10373

To appear in: $\quad B C P$

Received date: $\quad$ 18-7-2009

Revised date: $\quad 30-10-2009$

Accepted date: $\quad$ 2-11-2009

Please cite this article as: Tcherniuk S, van Lis R, Kozielski F, Skoufias DA, Mutations in the human kinesin Eg5 that confer resistance to monastrol and STrityl-L-Cysteine in tumor derived cell lines., Biochemical Pharmacology (2008), doi:10.1016/j.bcp.2009.11.001

This is a PDF file of an unedited manuscript that has been accepted for publication. As a service to our customers we are providing this early version of the manuscript. The manuscript will undergo copyediting, typesetting, and review of the resulting proof before it is published in its final form. Please note that during the production process errors may be discovered which could affect the content, and all legal disclaimers that apply to the journal pertain. 
Mutations in the human kinesin Eg5 that confer resistance to monastrol and S-Trityl-L-Cysteine in tumor derived cell lines.

\author{
Sergey Tcherniuk ${ }^{1,2, *}$, Robert van Lis ${ }^{1,3, *}$, Frank Kozielski ${ }^{4}$, Dimitrios A. Skoufias ${ }^{1 \#}$
}

${ }^{1}$ Institut de Biologie Structurale, (CEA-CNRS-UJF) 41 rue Jules Horowitz, 38027 Grenoble Cedex 01, France,

2 current address (CNRS - CRBM 1919, Route de Mende, 34293 Montpellier, Cedex 5, France),

3 current address (Bioénergétique et Ingénierie des Protéines, IFR88, CNRS, 13402 Marseille, France),

${ }^{4}$ The Beatson Institute for Cancer Research, Switchback Road, Bearsden, Glasgow G61 1BD, UK,

* Both authors contributed equally

\# Corresponding author:

Dimitrios A.Skoufias, $\mathrm{PhD}$

Institut de Biologie Structurale, J.P.Ebel

Laboratoire des Protéines du Cytosquelette

41 rue Jules Horowitz

Grenoble 38027 Cedex 1

France

Tel 33438789616

Fax 33438785494

dimitrios.skoufias@ibs.fr

Running title: 
Eg5 resistance to monastrol and STLC

\section{Abstract}

The kinesin Eg5 plays an essential role in bipolar spindle formation. A variety of structurally diverse inhibitors of the human kinesin Eg5, including monastrol and STLC, share the same binding pocket on Eg5, composed by helix $\alpha 2 /$ loop L5, and helix $\alpha 3$ of the Eg5 motor domain. Previous biochemical analysis in the inhibitor binding pocket of Eg5 identified key residues in the inhibitor binding pocket of Eg5 that in the presence of either monastrol or STLC exhibited ATPase activities similar to the untreated wild type Eg5. Here we evaluated the ability of fulllength human Eg5 carrying point mutations in the drug binding pocket to confer resistance in HeLa and U2OS cells to either monastrol or STLC, as measured by the formation of bipolar spindles. Both transfected cells expressing wild-type Eg5 and untransfected cells were equally sensitive to both inhibitors. Expression of Eg5 single point mutants R119A, D130A, L132A, I136A, L214A and E215A conferred significant resistance to monastrol. Certain mutations inducing monastrol resistance such as R119A, D130A and L214A also conferred significant resistance to STLC. For the first time at a cellular level, the propensity of selected Eg5 point mutants to confer drug resistance confirms the target specificity of monastrol and STLC for Eg5. These data also suggest a possible mechanism by which drug resistance may occur in tumors treated with agents targeting Eg5.

Keywords: Eg5, mitosis, monastrol, STLC, drug, resistance 


\section{Introduction}

The human kinesin Eg5 (also known as KSP, or Kif11), a member of the kinesin-5 family, is essential for the formation of a bipolar spindle in dividing cells [1]. Eg5 is a homotetrameric motor formed by the antiparallel arrangement of two dimers [2]. The Eg5 tetramer has the ability to crosslink antiparallel microtubules emanating from the two centrosomes at G2/M. Through its plus-end directed microtubule-based motor activity Eg5 is capable of generating outward forces which contribute to the separation of the two centrosomes, a process which is essential for the successful establishment of a bipolar spindle during mitosis [3, 4].

The success of the tubulin targeting agents (such as vinca alkaloids and taxanes) led to acceptance of the hypothesis that drugs targeting other proteins of the mitotic spindle may be a rational strategy to combat tumor growth with the advantage of having less side effects $[5,6]$. Due toEg5's essential function in mitosis, it is now an important target for the development of inhibitors that have antimitotic activity [7-9]. Following the discovery of monastrol [10], the first identified Eg5 inhibitor, a plethora of structurally related and unrelated inhibitors of Eg5 have been recently identified [11-13]. Earlier, we have described STLC as an Eg5-specific inhibitor which proved more potent than monastrol [14]. Chemical analogues of STLC have also been recently evaluated as potential inhibitors $[15,16]$. STLC, like monastrol and most if not all of the known Eg5 inhibitors, also inhibits mitosis, promoting the characteristic mono-astral spindle phenotype [10,17]. Induction of aberrant mitosis in tumor cells leads to consequent mitotic arrest, which is often followed by apoptotic cell death $[18,19]$. The fact that Eg5 inhibition in cancer cells results in a prolonged arrest in mitosis and an increased incidence of apoptosis has validated the apoptotic properties of Eg5 inhibitors [19-23].

Unfortunately, similar to the case for kinase inhibitors, highly selective chemical inhibitors of motor proteins are difficult to develop because all members of the kinesin superfamily share a conserved motor domain [24]. Thus, the issue of target specificity is of great importance for inhibitors targeting Eg5. Structural analyses of the interaction between the Eg5 motor domain and various inhibitors such as monastrol, its analogues and other 
inhibitor scaffolds have been recently carried out [25-29]. These ligands bind in a unique pocket in the Eg5 motor domain formed by the secondary elements helix $\alpha 2 /$ loop L5 (Tyr125-Glu145) and helix $\alpha 3$ (Ile202-Leu227) (shown in Fig. 1A). Loop 5 is part of an inhibitor binding pocket, that can accommodate different classes of inhibitors that can interact in a largely similar way. Drug binding induces allosterically changes in the mechanochemical cycle of the motor domain resulting in a slower ADP release rate from the active site, thus affecting the binding of Eg5 to microtubules [30-32]. Previous studies using hydrogen-deuterium exchange and mass spectrometry coupled with directed mutagenesis on the motor domain of human Eg5 showed that monastrol and STLC both bind to the same pocket [33,34]. Relatively subtle mutations in the binding pocket can confer resistance to drug binding without abolishing enzymatic function. In the presence of monastrol, and (for certain mutants) STLC, the basal and microtubule stimulated ATPase activities of the mutants were similar or diminished by up to a factor of 8 and their $\mathrm{K}_{\mathrm{M}}$ values for microtubules were increased when compared to those of the wild type Eg5 motor domain [34]. However, this purely in vitro study left unanswered questions including i) whether the mutants could confer resistance to anti-Eg5 agents in cell-based assays and ii) whether a compromised ATPase activity as a result of mutations in the catalytic core of the Eg5 motor domain would inhibit bipolar spindle formation in cells.

In this report here we introduced 10 point mutations in the allosteric drug binding pocket of the motor domain of full length Eg5 (Fig. 1B), and following transient transfection of HeLa cells we tested whether indeed these mutants could confer resistance to two chemically distinct inhibitors, monastrol or STLC. We identified mutations that were capable of conferring resistance to either monastrol or STLC, and certain of these mutations were consequential for both drugs. We discuss the significance of the results with reference to drug binding specificity as well as the drug resistance that may be encountered during chemotherapy using drugs that target the inhibitor binding site of Eg5. 


\section{Materials and methods}

\subsection{Cloning of point mutations and transfection}

The QuikChange kit (Stratagene, La Jolla, CA ) was used to introduce point mutations by alanine-scanning mutagenesis in the drug binding pocket (indicated in Fig. 1B) directly into the Eg5-pRcCMV plasmid coding for the native full-length Eg5 fused to a N-terminal myc-tag [1]. The sequences of the primers used and the unique restriction sites introduced are noted in Table 1 of supplemental material section. For clone selection purposes, a unique restriction site was introduced in addition to the point mutation by silent mutagenesis. Positive clones were subsequently confirmed by DNA sequencing.

\subsection{Cell culture, transient transfection, and immunofluorescence microscopy}

HeLa and U2OS cells were grown in D-MEM (Gibco BRL; Paisley, UK), suplemented with $10 \%$ fetal calf serum (Hyclone) on poly-D-lysine (Sigma-Aldrich, Saint Louis Missouri)-coated 12-mm-diameter glass coverslips up to confluence $70-80 \%$ on the day of transfection. Transfection of cells with the different plasmids was carried out using Lipofectamine 2000 (Invitrogen, Carlsbad, CA) according to the manufacture's protocol. Twenty four hours after transfection cells were exposed for $8 \mathrm{~h}$ to $100 \mu \mathrm{M}$ monastrol or $5 \mu \mathrm{M}$ STLC (Novabiochem, Merck KGaA, Darmstadt, Germany), both from $50 \mathrm{mM}$ stocks in DMSO) at $37^{\circ} \mathrm{C}$. Cells were then fixed by incubation in $2 \%$ paraformaldehyde $\left(20 \mathrm{~min}\right.$ at $\left.37^{\circ} \mathrm{C}\right)$ and permeabilized with $0.2 \%$ Triton X-100 in PBS for 3 min before being incubated with primary and stained with secondary antibodies. Expressed wild type and mutants of Eg5 were detected by a mouse monoclonal anti-myc antibody (Covance, Berkeley, CA) used at 200-fold dilution. TPX2 spindle protein was detected by rabbit polyclonal anti-TPX2 (Bethyl Laboratories, Motgomery, TX) antibodies at 400-fold dilution. The FITC-conjugated goat anti-mouse and goat Cy3-conjugated anti-rabbit secondary antibodies were used at 250-fold dilution and were purchased from Jackson (The Jackson Laboratory, West Grove, PA). DNA was detected either by incubation with $0.2 \mu \mathrm{g} / \mathrm{ml}$ propidium iodide in PBS for 5 min following incubations with 
secondary antibodies or with 4',6-Diamidino-2-phenylindole dihydrochloride (DAPI) with the VECTASHIELD (Vector Laboratories Inc, Burlingame, CA) mounting medium.

Images were collected with an MRC-600 Laser Scanning Confocal apparatus (BioRAD Laboratories) coupled to a Nikon Optiphot microscope, and an inverted Olympus IX81 epifluorescence motorized microscope equipped with a motorized piezo stage (Ludl Electronic Products, USA) and a Retiga-SRV CCD camera (QImaging, ) driven by VOLOCITY software (Improvision Ltd, UK) with a binning of 1, using a PlanApo 60x NA1.42 objective (Olympus). Images were processed in Photoshop version 7.0 (Adobe) and assembled in CANVAS version 8.0 (Denaba Systems).

Mitotic cells from three different series of transfection experiments were scored based on their phenotypes, myc-positive or myc-negative (at least 100 cells of each phenotype) and scored as bipolar or monoastral. The percentage of bipolar spindles myc-positive and myc-negative were then determined. Student's t-test analysis was carried out in order to evaluate the significance of the observed differences in the means of bipolar spindle formation resulting from expression of $\mathrm{Eg} 5$ mutants compared to wild-type $\mathrm{Eg} 5$ in the presence of monastrol or STLC. We classified a difference as being significant if the calculated t-value was equal or higher than tabulated tvalues at $p \leq 0.05$ ( $\geq 95 \%$ confidence), and as partial significant at $p \leq 0.1$ ( $\geq 90 \%$ confidence). In order to test whether undetectable levels of transfected Eg5 mutants (classified as myc-negative) can induce partial resistance to the drugs, manifested by an increase in the percentage of bipolar spindles in myc-negative cells in the total mycnegative population, we carried out correlation analysis of the means between the percentage of bipolar mycpositive and bipolar myc-negative cells transfected with Eg5 mutants. The calculated correlation coefficient was then evaluated with a table of correlation coefficients.

\subsection{Cell extracts and immunoblotting}


Twenty-four hours after transfection, cells were harvested and lysed in $50 \mathrm{mM}$ Tris- $\mathrm{HCl}, \mathrm{pH}$ 7.4, $250 \mathrm{mM}$ $\mathrm{NaCl}, 5 \mathrm{mM}$ EGTA, 0.1\% NP-40, supplemented with protease and phosphatase inhibitors, for 30 min on ice. Equal protein concentration of the different lysates were loaded onto polyacrylamide gels and then transferred to nitrocellulose. Expressed Eg5 was detected by a mouse anti-myc monoclonal antibody; endogenous Eg5 was detected with a polyclonal antibodies raised against the C-terminus of human Eg5 [1]; actin was detected by a polyclonal rabbit anti-pan-Actin antibody (Cell Signaling Technology Inc, Danvers, MA). Nitrocellulose sheets were then incubated with HRP-conjugated goat anti-mouse and anti-rabbit IgG secondary antibodies. Proteinantibody complexes were detected by enhanced chemiluminescence (Pierce Biotechnology, Rockford, IL, ). 


\section{Results}

\subsection{Expression of Eg5 point mutants in cells}

Single point mutations were introduced in the inhibitor binding pocket of a N-terminal myc tagged human Eg5 using an alanine-scanning mutagenesis strategy [34]. Previously, in vitro kinetic analysis of each of the motor domain mutants has shown that the mutations did not significantly alter the microtubule binding properties of the motor domain but indeed could influence the binding of either monastrol or STLC to Eg5 [34].

Following transient transfections of HeLa cells we verified by immunoblot analysis that each point mutant was expressed at approximately equivalent levels (Fig. 2A). In addition, the expressed Eg5 mutants were also capable of localizing to bipolar spindles in a diffuse manner, and in a localization pattern very similar to that observed by indirect immunofluorescence microscopy of tagged wild type Eg5 (Fig. 2B). Similar diffused spindle staining was observed for endogenous Eg5 using a C-terminal antibody to Eg5 (data not shown) as previously reported $[1,35]$. In the course of short term transient transfections there were no defects in bipolar spindle formation and function. Expression of wild type or mutant Eg5 did not induce any changes in bipolar spindle formation. In unsynchronized cell cultures and $24 \mathrm{~h}$ after transfection, the percentage of bipolar metaphase spindles of myc positive cells were comparable to that of untransfected (myc negative) cells, $50 \%$ vs $48 \%$, respectively. We have to note that although transient transfections in HeLa cells were successful in the period of $36 \mathrm{~h}$, we were unable to raise permanently transfected clones expressing either wild type or any of the Eg5 mutants. One possible explanation for our inability to raise stable expressing clones may come from the recent observation in the mouse model showing that overexpression of Eg5 resulted in tumorogenesis which was associated with chromosome instability due to perturbations of mitotic spindle assembly [36]. 


\subsection{Induction of resistance to monastrol in cells following expression of Eg5 point mutants}

Having established that the point mutants could be expressed and localized to bipolar spindles of transiently transfected cells, cells were then exposed to either monastrol or STLC, at inhibitor concentrations that induce maximal inhibitory effects as determined by the induction of monoastral spindles (100 $\mu \mathrm{M}$ and $5 \mu \mathrm{M}$, respectively). Untransfected control cells exposed to these concentrations arrest in mitosis with a monoastral spindle phenotype (Fig. 3A). We hypothesized that if the introduced Eg5 point mutants are capable of conferring resistance to monastrol or STLC, then cells expressing Eg5 mutants (detected by anti-myc) would be rescued in the presence of the drugs and capable of proceeding through mitosis and form normal bipolar spindles whereas untransfected cells (myc-negative cells) would be drug sensitive showing the characteristic monoastral spindle phenotype. Spindle morphology was determined by staining the spindles with an antibody against TPX2, a mitosis specific microtubule associated protein [37] (Fig. 3A).

Following exposure to monastrol $(100 \mu \mathrm{M}$, for $8 \mathrm{~h})$ the vast majority of mitotic spindles of cells expressing wild type Eg5 were monoastral (Fig. 3A). There were only $9.8 \pm 4.2 \%$ of myc-positive (myc+) bipolar spindles vs $11.2 \pm 4.9 \%$ of bipolar myc-negative (myc-) spindles; in untransfected cells there were only $9.4 \pm 2.7 \%$ bipolar spindles after similar monastrol treatment. This suggested that overexpression of wild type $\operatorname{Eg} 5$ did not change the sensitivity of cells to monastrol (Fig. 3 panels B and C). As observed for the wild type, the expression of V210A and Y211A Eg5 mutants did not alter the sensitivity of expressing cells to the drug (Fig. 3 panels B and C).

However, the expression of certain Eg5 mutants in the drug binding pocket conferred significant resistance to monastrol. For example expression of D130A and L214A Eg5 mutants resulted in $48 \%$ and $61 \%$ of mycpositive bipolar spindles, respectively (Fig. 3B). As expected, under the same conditions the non-expressing cells (myc- cells) were still sensitive to monastrol to the same degree as cells that were not transfected with $8.38 \%$ and 13.53\% (myc- bipolar spindles), respectively. Statistical analysis of the data by t-student's test of the data (suppl. material Table 2) confirmed that the expressed Eg5 mutants R119A, D130A, L132A, I136A, L214A and E215A 
conferred significant resistance to monastrol (with higher than 95\% confidence) when compared to the expression of wild type Eg5. Mutant V210A, conferred only partial resistance (90\% confidence). In contrast, expressed wild type, and mutants, W127A and R221A mutants and did not confer any significant resistance to monastrol.

The possibility existed that low expression of the mutants, at levels not detectable by indirect immunofluorescene microscopy, may have been also capable of inducing resistance to monastrol. However, if that were the case this would have been manifested in an increase in the percentage of bipolar spindles in myc-negative cells in the total myc-negative population. However, the percentage of myc-negative cells with bipolar spindles in the presence of monastrol remained at very low levels, equivalent to those obtained with either control cells or with the cells not transfected with wild type Eg5 (Fig. 3B). Furthermore, there was no correlation between the percentage of mitotic cells with bipolar spindles in cells expressing Eg5 mutants (myc+ cells) exposed to monastrol with an increase in the percentage of bipolar spindles in cells, in which there was no detectable expression of Eg5 mutants (myc- cells) (suppl material Table 3).

\subsection{Induction of resistance to STLC in cells following expression of Eg5 point mutants}

The same experimental protocol used in the monastrol study to determined whether the Eg5 point mutants could confer resistance to cells exposed to STLC, a chemically distinct inhibitor of Eg5 that is also thought to bind to the same inhibitor binding pocket in the motor domain as monastrol [29, 33, 34, 38].

We examined mitotic cells following treatment with STLC $(5 \mu \mathrm{M})$ during an $8 \mathrm{~h}$ period and determined that the majority of untransfected control cells (WT myc-) and Eg5-WT cells (WT myc+) had aberrant mitosis characterized by monoastral spindles (Fig. 4A). Cells exhibiting either bipolar or monastral spindles (Fig. 4A) in the presence of STLC were then quantitated in cell cultures expressing the different Eg5 point mutants. Statistically significant resistance (!95\% confidence) to STLC was observed in transfected cell expressing Eg5 carrying the mutations R119A, D130A, L214A, or R221A, whereas the mutation W127A conferred only a moderate resistance 
(!90\% confidence). Mutations L132A, I136A, V210A, Y211A or E215A did not confer any resistance to STLC

(Fig. 4B; suppl. Material Table 5). As was the case for monastrol, there was no correlation between bipolar spindle formation in cells expressing Eg5 mutants (myc+ cells) exposed to STLC and bipolar spindle formation in cells in which there was no detectable expression of Eg5 mutants (myc- cells) (Fig. 4C; suppl. Material Table 6).

\subsection{Induction of resistance to monastol and STLC in U2OS cells following expression of Eg5 point mutants}

We also addressed the question of whether resistance after overexpression of Eg5 point mutants is limited to HeLa cells or can be extended to other human tumor cell lines. For this purpose, U2OS cells were transfected with the two strongly resistant mutants D130A and L214A (see Fig. 3 and 4). In preliminary experiments U2OS were monastrol and STLC sensitive at the concentrations used in our studies (data not shown). Expression of D130A and L214A Eg5 mutants in U2OS cells conferred strong resistance to both drugs (Fig. 5). Cells expressing either of these mutants in the presence of monastrol or STLC were able to form normal bipolar spindles whereas the spindles in cells expressing wild type Eg5 exhibited the monoastral phenotype characteristic of Eg5 inhibition (Fig. 5A). Quantitation of the myc-positive spindles in the presence of monastrol showed that close to $69 \%$ of the spindles were bipolar for both mutants. Similarly, in the presence of STLC, $77 \%$ and $75 \%$ of the myc-positive spindles were bipolar when D130A and L214A mutants of Eg5 were overexpressed, respectively. 


\section{Discussion}

The data presented here demonstrate that mutations in the induced fit binding pocket of Eg5 can confer drug resistance in cells to inhibitors that are known to bind to this pocket, such as monastrol and STLC. We have to note that in our study the definition of resistance is limited to the ability of cells to separate the duplicated centrosomes during mitotic entry in the presence of the inhibitors. The ability of the mutants to allow the formation of a bipolar spindle in cells exposed to Eg5 inhibitors confirms for the first time at the cellular level that indeed Eg5 is the sole target of the two inhibitors in mitosis. Furthermore, as cells exhibited resistance to two chemically distinct inhibitors on overexpression of the same mutants, the data strongly suggest that development of drug resistance in tumor cells treated with anti-Eg5 agents may be a real prospect.

Monastrol and STLC have been characterized as Eg5 specific inhibitors exhibiting antimitotic activity. However, as is the case for any other inhibitor, the issue of drug-target specificity is a very difficult problem to resolve. In the cases of monastrol and STLC, their specificity for Eg5 was approached by testing the ability of the drugs to inhibit other kinesins that may be involved in mitosis. Indeed, with in vitro biochemical studies both drugs proved to be specific for Eg5 and did no inhibit a panel of other mitotic motor proteins. However, the list of the motor proteins tested was not exhaustive because there are about 45 kinesins in the human genome and only about 12 have been tested so far. In addition, the elucidation of the structure of the Eg5-inhibitor complexes showed that all allosteric drugs tested so far bind to the motor domain in a pocket formed by helix $\alpha 2 /$ loop L5 and helix $\alpha 3$. The large loop L5 is unique to Eg5, because in all other members of the kinesin superfamily this loop is considerably shorter. Despite these results the issue of specificity for Eg5 drug binding in cells is still not answered. Our report attempts to answer this question at the cellular level. Our approach was to test the ability of point mutants in the inhibitor binding pocket to confer resistance to either monastrol or STLC when expressed in tumor derived cells. These studies complemented previous biochemical in vitro studies that showed that the same 
mutations resulted in Eg5 motor domains exhibiting ATPase activities similar or close to that of wild type when exposed to both drugs [34]. The cumulative in vitro biochemical and cell-based results are presented in Table 1.

Overall the drug resistance observed by cell-based assays were in agreement with the biochemical assays reported earlier. Mutations in residues R119, D130, or L214 conferred significant resistance to two chemically distinct inhibitors, monastrol and STLC. The ability of the mutants to form a normal bipolar spindle in the presence of either monastrol or STLC argues strongly that the antimitotic function of both inhibitors is related to their binding to $\mathrm{Eg} 5$ and that $\mathrm{Eg} 5$ is the sole protein target in cells in mitosis. The fact that resistance observed with multiple single point mutations suggests that there is not a single amino acid in the pocket that is essential for drug mediated inhibition of Eg5, but rather, there are many different residues within the induced fit pocket of Eg5 that are equally important for drug binding. Indeed, there may be additional residues not tested, that could also prove important for binding and /or for the allosteric inhibition of Eg5.

R119 is located in the entrance of the binding pocket and its side chain undergoes a major shift in order to accommodate binding of monastrol. In agreement with the observed resistance in cells imposed by R119A, earlier biochemical assays have shown that the R119A mutation changes STLC from a tight-binding inhibitor to a conventional inhibitor with lower affinity for Eg5. Similarly, the D130A mutation abolished monastrol binding and decreased the affinity constant for STLC. Furthermore L214, which conferred the highest resistance in cells, was previously identified as a key residue for both the inhibition by monastrol and STLC [34]. In the crystal structure of the complex the side chains of D130 and L214 point towards monastrol and L214 undergoes a shift in order to accommodate monastrol binding in the binding pocket. The observed differences in the drug resistance imposed by the I136A mutant (located in the core of the binding pocket) in cell-based assays were also observed in the binding assays in vitro. I136A diminished monastrol binding but had no impact on STLC binding.

The observed resistance in cells conferred by L132A and E215 were two notable differences between cellbased and in vitro biochemical assays. L132 and E215 are located in loop L5 and helix $\alpha 3$, respectively and 
exposed to the solvent and were not effective to alter monastrol or STLC binding to Eg5 in vitro. Both mutants were shown previously to not alter the inhibitory activities of either monastrol or STLC and in cells did not confer any resistance to STLC. However, when expressed in cells, L132A and E215A conferred resistance to monastrol. One possible explanation for this discrepancy may be that these two residues in the cellular context may be in a different conformation possibly through interaction with a yet unidentified binding protein that may change the overall conformation of the binding pocket making it less accommodating for monastrol.

Although W127 undergoes a shift of $9 \AA$ upon inhibitor binding and is an important component of the binding pocket, its substitution by Ala conferred only partial resistance both in cell-based assays for both monastrol and STLC as well as in biochemical assays in vitro.

The partial resistance in cells to monastrol by the V210A mutant may be explained by the in vitro observations that monastrol is still able to bind to the V210 mutant although the mutation increased the $\mathrm{IC}_{50}$ value and the inhibitor constant. Even though V210 does not interact directly with monastrol it may contribute to the overall rigidity of the binding pocket. Furthermore, the partial resistance or lack of resistance to monastol and STLC conferred in cells by R221A mutant may be explained by the fact that the R221 residue is located deep in the binding pocket.

Another interesting outcome of the results presented here is that certain mutants of Eg5 can confer significant resistance to either monastrol (R119A, D130A, L132A, I136A, L214A and E215A) or STLC (R119A, D130A and L214A) when expressed in cells in the presence of endogenous Eg5, which is drug sensitive. Eg5 from different species have been shown to function as tetramers $[2,39,40]$ and thus under our experimental conditions Eg5 should be a promiscuous homotetramer composed of endogenous drug sensitive Eg5 and ectopically expressed drug resistant Eg5. If this is the case, then the observed resistance to either monastrol or STLC raises questions concerning the mode of displacement/processivity of Eg5 along microtubules. Indeed, based on recent in vitro motility studies of an artificial kinesin-1-Eg5 heterodimer it was proposed that Eg5 may work in a predominantly 
nonprocessive manner and that there might very little motor head-head coordination in the Eg5 tetramer [41]. According to this model an Eg5 tetramer composed of drug sensitive and drug resistant Eg5 motor heads will be able to produce productive forces even in the presence of inhibitors, as we report here.

Drug resistance is major problem encountered in cancer chemotherapy and can arise by many different mechanisms. Tumor cells for example can develop resistance to antimitotic inhibitors that target tubulin such as taxanes and vinca alkaloids [42] through mutations in the drug binding site (i.e. $\beta$ tubulin) [43, 44] or by overexpression of the drug efflux pump, P-glycoprotein [45]. The ability to confer drug resistance by introducing mutations in the induced fit binding pocket of Eg5 raises the possibility that such mutations may be encountered in tumors during chemotherapy if Eg5 inhibitors were to enter the clinic. In support of our results it has been reported that Ispinesib-resistant cancerous cells were found to harbor a D130V substitution located in loop L5 of Eg5 [46]. Our data reported here have validated residue D130 as a key residue in monastrol and STLC resistance in cells. New Eg5 inhibitors are appearing rapidly and therefore the development of well defined drug resistant cell lines would be a useful tool in testing their efficacy, alone or in combination with other inhibitors in order to circumvent drug resistance in the clinic. 


\section{Acknowledgements}

This work has been funded by grants from ARC (Association pour la Recherche sur le Cancer, contract number 3973) and Cancer Research UK. S.T. was supported by a post-doctoral fellowship from ARC, R.vL. held a postdoctoral grant from the Rhône-Alpes region (reference 0501891003). We thank Chris Gray for his comments on the manuscript. 


\section{References}

[1] Blangy A, Lane HA, d'Herin P, Harper M, Kress M, Nigg EA. Phosphorylation by p34cdc2 regulates spindle association of human Eg5, a kinesin-related motor essential for bipolar spindle formation in vivo. Cell 1995;83:1159-69.

[2] Kashina AS, Baskin RJ, Cole DG, Wedaman KP, Saxton WM, Scholey JM. A bipolar kinesin. Nature $1996 ; 379: 270-2$.

[3] Sharp DJ, Rogers GC, Scholey JM. Microtubule motors in mitosis. Nature 2000;407:41-7.

[4] Kapitein LC, Peterman EJ, Kwok BH, Kim JH, Kapoor TM, Schmidt CF. The bipolar mitotic kinesin Eg5 moves on both microtubules that it crosslinks. Nature 2005;435:114-8.

[5] Jordan MA, Wilson L. Microtubules as a target for anticancer drugs. Nat Rev Cancer 2004;4:253-65.

[6] Bhalla KN. Microtubule-targeted anticancer agents and apoptosis. Oncogene 2003;22:9075-86.

[7] Bergnes G, Brejc K, Belmont L. Mitotic kinesins: prospects for antimitotic drug discovery. Curr Top Med Chem 2005;5:127-45.

[8] Sakowicz R, Finer JT, Beraud C, Crompton A, Lewis E, Fritsch A, et al. Antitumor activity of a kinesin inhibitor. Cancer Res 2004;64:3276-80.

[9] Schmidt M, Bastians H. Mitotic drug targets and the development of novel anti-mitotic anticancer drugs. Drug Resist Updat 2007;10:162-81.

[10] Mayer TU, Kapoor TM, Haggarty SJ, King RW, Schreiber SL, Mitchison TJ. Small molecule inhibitor of mitotic spindle bipolarity identified in a phenotype-based screen. Science 1999;286:971-4.

[11] Sarli V, Giannis A. Targeting the kinesin spindle protein: basic principles and clinical implications. Clin Cancer Res 2008;14:7583-7.

[12] Huszar D, Theoclitou ME, Skolnik J, Herbst R. Kinesin motor proteins as targets for cancer therapy. Cancer Metastasis Rev 2009. 
[13] Zhang Y, Xu W. Progress on kinesin spindle protein inhibitors as anti-cancer agents. Anticancer Agents Med Chem 2008;8:698-704.

[14] DeBonis S, Skoufias DA, Lebeau L, Lopez R, Robin G, Margolis RL, et al. In vitro screening for inhibitors of the human mitotic kinesin Eg5 with antimitotic and antitumor activities. Mol Cancer Ther 2004;3:107990.

[15] Debonis S, Skoufias DA, Indorato RL, Liger F, Marquet B, Laggner C, et al. Structure-activity relationship of S-trityl-1-cysteine analogues as inhibitors of the human mitotic kinesin Eg5. J Med Chem 2008;51:111525.

[16] Ogo N, Oishi S, Matsuno K, Sawada J, Fujii N, Asai A. Synthesis and biological evaluation of L-cysteine derivatives as mitotic kinesin Eg5 inhibitors. Bioorg Med Chem Lett 2007;17:3921-4.

[17] Skoufias DA, DeBonis S, Saoudi Y, Lebeau L, Crevel I, Cross R, et al. S-trityl-L-cysteine is a reversible, tight binding inhibitor of the human kinesin Eg5 that specifically blocks mitotic progression. J Biol Chem 2006;281:17559-69.

[18] Gascoigne KE, Taylor SS. Cancer cells display profound intra- and interline variation following prolonged exposure to antimitotic drugs. Cancer Cell 2008;14:111-22.

[19] Shi J, Orth JD, Mitchison T. Cell type variation in responses to antimitotic drugs that target microtubules and kinesin-5. Cancer Res 2008;68:3269-76.

[20] Kozielski F, Skoufias DA, Indorato RL, Saoudi Y, Jungblut PR, Hustoft HK, et al. Proteome analysis of apoptosis signaling by S-trityl-L-cysteine, a potent reversible inhibitor of human mitotic kinesin Eg5. Proteomics 2008;8:289-300.

[21] Tao W, South VJ, Zhang Y, Davide JP, Farrell L, Kohl NE, et al. Induction of apoptosis by an inhibitor of the mitotic kinesin KSP requires both activation of the spindle assembly checkpoint and mitotic slippage. Cancer Cell 2005;8:49-59. 
[22] Marcus AI, Peters U, Thomas SL, Garrett S, Zelnak A, Kapoor TM, et al. Mitotic kinesin inhibitors induce mitotic arrest and cell death in Taxol-resistant and -sensitive cancer cells. J Biol Chem 2005;280:11569-77.

[23] Orth JD, Tang Y, Shi J, Loy CT, Amendt C, Wilm C, et al. Quantitative live imaging of cancer and normal cells treated with Kinesin-5 inhibitors indicates significant differences in phenotypic responses and cell fate. Mol Cancer Ther 2008;7:3480-9.

[24] Dagenbach EM, Endow SA. A new kinesin tree. J Cell Sci 2004;117:3-7.

[25] Garcia-Saez I, DeBonis S, Lopez R, Trucco F, Rousseau B, Thuery P, et al. Structure of human Eg5 in complex with a new monastrol-based inhibitor bound in the R configuration. J Biol Chem 2007;282:97407.

[26] Yan Y, Sardana V, Xu B, Homnick C, Halczenko W, Buser CA, et al. Inhibition of a mitotic motor protein: where, how, and conformational consequences. J Mol Biol 2004;335:547-54.

[27] Zhang B, Liu JF, Xu Y, Ng SC. Crystal structure of HsEg5 in complex with clinical candidate CK0238273 provides insight into inhibitory mechanism, potency, and specificity. Biochem Biophys Res Commun 2008;372:565-70.

[28] Cox CD, Breslin MJ, Mariano BJ, Coleman PJ, Buser CA, Walsh ES, et al. Kinesin spindle protein (KSP) inhibitors. Part 1: The discovery of 3,5-diaryl-4,5-dihydropyrazoles as potent and selective inhibitors of the mitotic kinesin KSP. Bioorg Med Chem Lett 2005;15:2041-5.

[29] Kaan HY, Ulaganathan V, Hackney DD, Kozielski F. An allosteric transition trapped in an intermediate state of a new kinesin-inhibitor complex. The Biochemical journal 2009.

[30] DeBonis S, Simorre JP, Crevel I, Lebeau L, Skoufias DA, Blangy A, et al. Interaction of the mitotic inhibitor monastrol with human kinesin Eg5. Biochemistry 2003;42:338-49.

[31] Maliga Z, Kapoor TM, Mitchison TJ. Evidence that monastrol is an allosteric inhibitor of the mitotic kinesin Eg5. Chem Biol 2002;9:989-96. 
[32] Krzysiak TC, Wendt T, Sproul LR, Tittmann P, Gross H, Gilbert SP, et al. A structural model for monastrol inhibition of dimeric kinesin Eg5. Embo J 2006;25:2263-73.

[33] Brier S, Lemaire D, Debonis S, Forest E, Kozielski F. Identification of the protein binding region of Strityl-L-cysteine, a new potent inhibitor of the mitotic kinesin Eg5. Biochemistry 2004;43:13072-82.

[34] Brier S, Lemaire D, DeBonis S, Forest E, Kozielski F. Molecular dissection of the inhibitor binding pocket of mitotic kinesin Eg5 reveals mutants that confer resistance to antimitotic agents. J Mol Biol 2006;360:360-76.

[35] Blangy A, Arnaud L, Nigg EA. Phosphorylation by p34cdc2 protein kinase regulates binding of the kinesin-related motor HsEg5 to the dynactin subunit p150. J Biol Chem 1997;272:19418-24.

[36] Castillo A, Morse HC, 3rd, Godfrey VL, Naeem R, Justice MJ. Overexpression of Eg5 causes genomic instability and tumor formation in mice. Cancer Res 2007;67:10138-47.

[37] Wittmann T, Wilm M, Karsenti E, Vernos I. TPX2, A novel xenopus MAP involved in spindle pole organization. J Cell Biol 2000;149:1405-18.

[38] Brier S, Lemaire D, DeBonis S, Kozielski F, Forest E. Use of hydrogen/deuterium exchange mass spectrometry and mutagenesis as a tool to identify the binding region of inhibitors targeting the human mitotic kinesin Eg5. Rapid Commun Mass Spectrom 2006;20:456-62.

[39] Cole DG, Saxton WM, Sheehan KB, Scholey JM. A "slow" homotetrameric kinesin-related motor protein purified from Drosophila embryos. J Biol Chem 1994;269:22913-6.

[40] Gordon DM, Roof DM. The kinesin-related protein Kip1p of Saccharomyces cerevisiae is bipolar. J Biol Chem 1999;274:28779-86.

[41] Kaseda K, Crevel I, Hirose K, Cross RA. Single-headed mode of kinesin-5. EMBO Rep 2008;9:761-5.

[42] Kavallaris M, Annereau JP, Barret JM. Potential mechanisms of resistance to microtubule inhibitors. Semin Oncol 2008;35:S22-7. 
[43] Giannakakou P, Sackett DL, Kang YK, Zhan Z, Buters JT, Fojo T, et al. Paclitaxel-resistant human ovarian cancer cells have mutant beta-tubulins that exhibit impaired paclitaxel-driven polymerization. J Biol Chem $1997 ; 272: 17118-25$.

[44] Kavallaris M, Tait AS, Walsh BJ, He L, Horwitz SB, Norris MD, et al. Multiple microtubule alterations are associated with Vinca alkaloid resistance in human leukemia cells. Cancer Res 2001;61:5803-9.

[45] Geney R, Ungureanu M, Li D, Ojima I. Overcoming multidrug resistance in taxane chemotherapy. Clin Chem Lab Med 2002;40:918-25.

[46] Luo L, Parrish CA, Nevins N, McNulty DE, Chaudhari AM, Carson JD, et al. ATP-competitive inhibitors of the mitotic kinesin KSP that function via an allosteric mechanism. Nat Chem Biol 2007;3:722-6. 


\section{Figure Legends}

Fig. 1. (A) Surface rendering plot of the motor domain of human Eg5 with $\mathrm{Mg}^{2+} \mathrm{ADP}$ and monastrol bound in the allosteric drug binding pocket (left panel). The different amino acid residues mutated in the binding pocket in the blow up were labeled with distinct colors for facilitating their identification). (B) Bar diagram of the human mitotic kinesin Eg5, containing the N-terminal motor domain, an internal $\alpha$-helical region predicted to form a discontinuous coiled-coil, and the C-terminal tail domain. The point mutations introduced by alanine scanning mutagenesis in the inhibitor binding pocket of the motor domain are indicated.

Fig. 2. Expression and localization of the Eg5 point mutants. (A) HeLa cells were transiently transfected with the various Eg5 point mutants and after $24 \mathrm{~h}$ total cell extracts were loaded on SDS-PAGE for immunoblot analysis using anti-myc for detection of the ectopically expressed $\mathrm{Eg} 5$, anti-Eg5 to compare the levels of overexpression relatively to endogenous Eg5 from untransfected control and anti-actin as protein loading control. (B) Transfected cells were fixed and stained for indirect immunoflourescence microscopy to detect expressed $\mathrm{Eg} 5$ using an antimyc monoclonal antibody (in green) and DNA stained with propidium iodide (red). Expressed wild type Eg5 and all point mutants of the drug binding pocket of Eg5 were diffusely distributed in the mitotic spindle.

Fig. 3. Resistance to monastrol of HeLa cells expressing Eg5 point mutants. (A) Transfected cells in the absence and presence of monastrol $(100 \mu \mathrm{M}$, for $8 \mathrm{~h})$ were fixed and stained for indirect immunoflourescence microscopy. Expressed Eg5 was detected using an anti-myc monoclonal antibody (green); the spindles were stained with antiTPX2, a spindle associated protein (red), and chromatin was stained with DAPI (blue). The presence of bipolar myc-positive and myc-negative bipolar and monoastral spindles were scored and the \% of bipolar spindles over the total spindles of each class were determined and plotted as bar graphs for myc+ bipolar spindles in panel (B) and 
for myc- in panel (C). Data were analyzed by student's t-test. Dark bars: significant resistance ( $\mathrm{p} \leq 0.05 ; 195 \%$ confidence). Gray bars: partial resistance ( $\mathrm{p} \leq 0.1 ; ! 90 \%$ confidence). Open bars: no resistance.

Fig. 4. Resistance to STLC of HeLa cells expressing Eg5 point mutants. (A) Transfected cells were exposed to STLC $(5 \mu \mathrm{M}$, for $8 \mathrm{~h})$ before being fixed and stained for indirect immunoflourescence microscopy. Expressed Eg5 was detected using an anti-myc monoclonal antibody (green); the spindle was stained with anti-TPX2 (red) and chromatin with DAPI (blue). The presence of bipolar myc-positive and myc-negative bipolar and monoastral spindles were scored and the $\%$ of bipolar spindles over the total spindles of each class were determined and plotted as bar graphs for myc+ bipolar spindles in panel (B), and for myc- in panel (C). Data were analyzed by student's t-test. Dark bars: significant resistance ( $\mathrm{p} \leq 0.05 ;$ ! $95 \%$ confidence). Gray bars: partial resistance $(\mathrm{p} \leq 0.1$; !90\% confidence). Open bars: no resistance.

Fig. 5. Resistance to monastrol and to STLC of U2OS cells expressing Eg5 point mutants. (A) Representative images of mitotic cells expressing either WT or D130A or L214A myc-tagged Eg5 exposed to either monastrol (100 $\mu \mathrm{M}$, for $8 \mathrm{~h}$; upper panels) or to STLC ( $5 \mu \mathrm{M}$, for $8 \mathrm{~h}$; lower panels). Expressed Eg 5 was detected by an antimyc monoclonal antibody (green), and spindles and chromatin were stained with anti-TPX2 (red) and DAPI (blue), respectively. (B) The presence of myc-positive bipolar and monoastral spindles were scored and the \% of myc-positive bipolar spindles over the total spindles counted were determined and plotted as bar graphs. Black bars: monastrol; Gray bars: STLC. 
Page 24 of 24 